Darwin ${ }^{13}$ suggested that our facial expressions have been conserved throughout evolution; that is, if we look carefully, we will see similar expressions among nonhuman animal species. The study by Susskind et al. ${ }^{1}$ suggests that it might be possible to find a biologically adaptive basis for all facial expressions. On the other hand, as long as a particular trait does not confer a disadvantage, it will also survive throughout evolution. It is therefore possible that the shape of some facial expressions confers a particular biological advantage, whereas others are either more random in their configuration or resulted from some nonbiological selection pressure.

For humans, even if a given facial expression originally evolved on the basis of one adaptive benefit, social evolution can assimilate it for its own purposes. It's fun to think about smiles in this context. How did baring a full mouth of teeth become the positive expression in our social world? Although Darwin thought he saw monkeys smiling ${ }^{15}$, if one looks carefully you eventually notice that there are no smiles between monkeys. There are playful faces, but no true smiles (S.E. Shelton, Wisconsin National Primate Research Center, personal communication). There is, however, a smile-like expression where the head tilts forward as the gaze is averted and the teeth are exposed by turning up the corners of the mouth. But, in the monkey world, this is no smile; this is a sign of fear, referred to as the fear grimace.
Ultimately, the fear grimace is a signal of submission. Subordinate monkeys offer it to dominant monkeys in appeasement. It's ironic that Darwin misinterpreted this expression as a true smile, as the fear grimace may well be the precursor of the human smile. For we often use smiles as a form of submission. As an example, imagine that you have a friend who thinks he's funnier than he actually is, but then his eleventh joke of the night is actually quite funny. You look down as you grin in a sort of, "Alright, you got me," gesture, a simple human form of submission.

What probably happened over time is that the originally submissive smile took on all kinds of newer meanings that were demanded by expanding social complexity. And now we find ourselves with an expression that indexes not only our own state of happiness, contentment, peace or even embarrassment, but also one that can embody what we want to say to others, in terms of acceptance, warmth or desire. The baring of teeth has socially evolved. More generally, this example demonstrates that the functional outcomes of biological and social evolution can coexist.

Facial expressions are powerful. They are succinct. And they are everywhere. Susskind et al. ${ }^{1}$ have given us much to consider in terms of understanding why we look the way we do in certain situations. Future research can address the relative biological versus social importance of various facial expressions. If there truly is ongoing social evolution of facial configurations, then we can only speculate about the future social pressures that will exist and of the new facial expressions to come, or of the ways in which existing expressions will be co-opted for new purposes. In the interim, your friends will make faces in situations that have relevance for the two of you. If you find yourself wondering if that face they just made was 'all about them' or 'all about you', know that it can simultaneously be about the both of you.

1. Susskind, J.M. et al. Nat. Neurosci. 11, 843-850 (2008).

2. Whalen, P.J. et al. Science 306, 2061 (2004).

3. Adolphs, R. et al. Nature 433, 68-72 (2005).

4. Gloor, P. The Temporal Lobe and Limbic System (Oxford University Press, New York, 1997).

5. Demos, K. et al. Cereb. Cortex (in the press).

6. Harrison, N.A. et al. Soc. Cogn. Affect. Neurosci. 1, 5-17 (2007).

7. Whalen, P.J. Curr. Dir. Psychol. Sci. 7, 177-186 (1998).

8. Kapp, B.S. et al. in The Amygdala (ed. Aggleton, J.P.) (Wiley-Liss, New York, 1992)

9. Phillips, M.L. et al. Nature 389, 495-498 (1997).

10. Krolak-Salmon, P. et al. Ann. Neurol. 53, 446-453 (2003).

11. Calder, A.J. et al. Nat. Neurosci. 3, 1077-1078 (2000).

12. Sprengelmeyer, R. et al. Brain 119, 1647-1665 (1996).

13. Anderson, A.K. et al. J. Neurosci. 23, 5627-5633 (2003).

14. Marsh, A.A. et al. Emotion 5, 119-124 (2005).

15. Darwin, C. The Expression of the Emotions in Man and Animals (John Murray, London, 1872).

\title{
Awake and asleep
}

Two-photon imaging in vivo has been used to monitor the activity of populations of cortical neurons. With some calcium dyes, these measurements have single-cell and single actionpotential resolution. However, previous experiments looking at the spatiotemporal interactions of a large population of neurons have only been carried out in anesthetized animals. Until now, the relationship between population activity in the anesthetized animal and the awake animal has remained unclear. A new study on page 749 by Greenberg and colleagues now provides direct evidence to bridge this gap. Their findings suggest that the neuronal activity patterns of awake animals cannot be predicted from activity patterns seen under anesthesia.

The authors imaged calcium transients in layer $2 / 3$ neurons in the visual cortex of awake, head-restrained rats, then anesthetized them and continued recording. In this way, they were able to measure the activity of the same neuronal population while the animals were awake and while they were anesthetized. The authors then used an algorithm that they had previously developed to identify action potential-evoked calcium transients based on the correspondence between the optical signal and the electrocorticogram. The algorithm was optimized using a separate set of data from simultaneous cell-attached electrophysiological

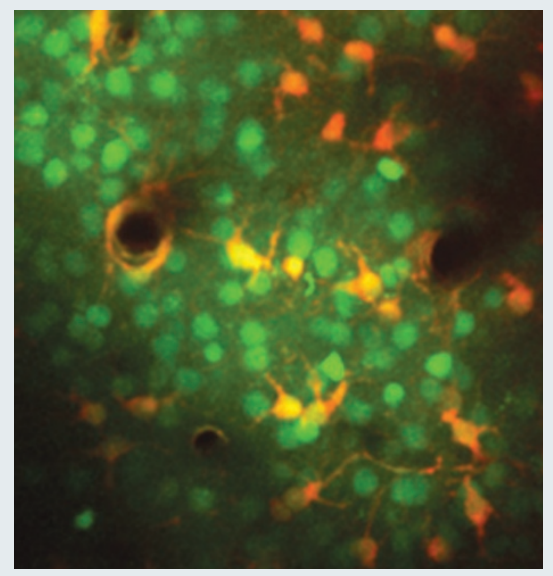
and optical recordings in anesthetized animals.

Neuronal firing rates were much lower in anesthetized animals than in the awake animals, although the firing rates of individual neurons were correlated in the two states. During both anesthesia and awake periods, action potential firing in a single neuron temporarily increased action potential firing in the surrounding population; this effect was stronger for some neurons than for others and was stronger during anesthesia. Although neurons that were closer together were more likely to be influenced the same way by the onset of anesthesia, correlations between pairs of neurons in the awake state were independent of the correlations in the anesthetized state. There was a higher correlation between neurons in the anesthetized state, and population synchrony was also greater in the anesthetized condition.

These results represent the first direct comparison between neural population activity in awake and anesthetized animals. They suggest that the local patterns of activity in awake animals will probably provide stronger inputs to their targets in layer 5 than in anesthetized animals. More generally, these findings raise the important caveat that the spatiotemporal structure of action potential firing in a population of neurons recorded in an awake animal can not be directly inferred from recordings of the same neurons under the influence of anesthesia.

Hannah Bayer 